\title{
Non-Alcoholic Fatty Liver Disease in Patients Attending the National Severe Insulin Resistance Service
}

Sarah M Leiter ${ }^{1}$, Alison Sleigh², Claire Adams ${ }^{1,3}$, Julie Harris ${ }^{1,3}$, David J Lomas ${ }^{4,5}$, Michael Allison ${ }^{6}$, Stephen O'Rahilly ${ }^{1,3}$, Robert Semple ${ }^{1,3}$, David Savage ${ }^{1,3}$, Anna Stears ${ }^{1,3}$

1 Metabolic Research Laboratories, University of Cambridge, Cambridge, UK, 2 Wolfson Brain Imaging Centre, University of Cambridge, Cambridge, UK 3 Wolfson Department of Diabetes and Endocrinology, Addenbrooke's Hospital, Cambridge, UK 4 Department of Radiology, University of Cambridge, Cambridge, UK 5 Department of Radiology, Addenbrooke's Hospital, Cambridge, UK 6 Department of Medicine, Addenbrooke's Hospital, Cambridge, UK

\section{Introduction}

The prevalence of non-alcoholic fatty liver disease (NAFLD) is greatly increased in patients with lipodystrophy and some but not all other causes of Severe Insulin Resistance (SIR). Although liver biopsy remains the definitive technique for diagnosis and staging of NAFLD, non-invasive techniques, such as magnetic resonance spectroscopy (MRS) and magnetic resonance imaging (MRI) are increasingly used to assess response to interventions such as leptin therapy. The National Severe Insulin Resistance Service is commissioned by the National Specialist Commissioning Team for patients from England with lipodystrophy and/or SIR. Selected patients attending the service, including all those receiving leptin therapy, are offered annual measurement of \% liver fat using either MRS or MRI.

\section{Patient population}

31 patients have received MR scans to date $(26 \mathrm{~F}, 5 \mathrm{M})$ median (range) age 35 (13-58) years, BMI 25.7 (15.8-37.8) $\mathrm{kg} / \mathrm{m}^{2} .13$ patients are taking leptin therapy. 26 have diabetes. None have an insulin receptor mutation.

\begin{tabular}{|c|c|} 
SIR Diagnosis & $\begin{array}{c}\text { Number of } \\
\text { patients }\end{array}$ \\
\hline
\end{tabular}

Familial Partial Lipodystrophy (FPLD) 19

Congenital Generalised Lipodystrophy (CGL) 4

Acquired Generalised Lipodystrophy (AGL) 2

Acquired Partial Lipodystrophy (APL)

2

SIR unknown cause

Table 1: SIR diagnoses in the 31 patients scanned

MR scanning techniques

Magnetic Resonance Spectroscopy: In 13/31 patients, \% liver fat was measured using proton magnetic resonance spectroscopy on a Siemens $3 T$ Verio MR scanner. A nonwater suppressed, respiratory gated, spectrum was obtained from a single voxel located within the posterior aspect of the right lobe of the liver (64 averages, TR $=7 \mathrm{~s}$ ). Liver fat was quantified by comparing the $\mathrm{CH} 2$ resonance at $1.3 \mathrm{ppm}$ with water at $4.7 \mathrm{ppm}$.

Magnetic Resonance Imaging: In 18/31 patients, \% liver fat was measured using in-phase and out of phase gradient-echo MRI with dual flip angles of 20 and 70 degrees corrected for $\mathrm{T}^{*}$ decay.

\section{Results}

$26 / 31(84 \%)$ of patients had a liver fat of $>5 \%$ (Table 2). However in two patients with biopsy proven fibrotic liver disease the \% liver fat was unexpectedly low $(4.0 \%$ and $6.0 \%$ respectively), (Figure 1b). There were significant correlations between \% liver fat and plasma bilirubin/ALT in patients receiving MRS but not $\mathrm{MRI}$. There were no significant correlations between \% liver fat and fasting triglycerides, $\mathrm{HbA1c}$ or BMI, but this is likely to be due to the relatively small number of patients (Table 3 ).

\begin{tabular}{|c|c|c|}
\hline & MRI & MRS \\
\hline Number of patients & 18 & 13 \\
\hline \% Liver fat (NR<5\%) & $9.5(7.0,15.0)$ & $22.6(10.5,36.8)$ \\
\hline $\begin{array}{l}\text { Bilirubin } \\
\text { (NR 0-17 } \mu \mathrm{mol} / \mathrm{L} \text { ) }\end{array}$ & $9.5(6.3,11.8)$ & $7.0(6.0,8.0)$ \\
\hline ALT (NR 0-50IU/L) & $47.0(29.3,55.3)$ & $30.5(22.5,70.0)$ \\
\hline ALP (NR 30-135IU/L) & $105.0(79.0,135.0)$ & $77.0(69.0,102.0)$ \\
\hline GGT (NR 0-33IU/L) & $51.0(28.0,85.0)$ & $33.0(26.0,88.0)$ \\
\hline $\begin{array}{l}\text { Fasting triglycerides } \\
\text { (NR }<1.7 \mathrm{mmol} / \mathrm{L} \text { ) }\end{array}$ & $2.4(1.6,3.6)$ & $2.5(1.6,6.3)$ \\
\hline $\mathrm{HbA1c}(\mathrm{mmol} / \mathrm{mol})$ & $69.0(46.0,77.0)$ & $53.0(43.0,75.0)$ \\
\hline BMI $\left(\mathrm{kg} / \mathrm{m}^{2}\right)$ & $26.1(25.1,28.8)$ & $25.7(21.4,31.1)$ \\
\hline Diabetes & $16(89 \%)$ & $10(77 \%)$ \\
\hline Leptin therapy & 7 (39\%) & $6(46 \%)$ \\
\hline
\end{tabular}

Table 2: Median (IQR) \% liver fat, BMI, liver function and metabolic blood tests in patients receiving MR scans (NR=normal range)

\begin{tabular}{|l|c|c|c|c|}
\hline & \multicolumn{2}{|c|}{ MRI } & \multicolumn{2}{c|}{ MRS } \\
\hline Bilirubin & $r$ & $p$ & $r$ & $p$ \\
\hline ALT & -0.33 & ns & 0.57 & $0.04^{*}$ \\
\hline GGT & 0.11 & ns & 0.58 & $0.05^{\star}$ \\
\hline Fasting triglycerides & 0.21 & ns & 0.44 & ns \\
\hline HbA1c (mmol/mol) & 0.04 & ns & 0.12 & ns \\
\hline BMI & 0.36 & ns & 0.31 & ns \\
\hline
\end{tabular}

Table 3: Spearman rank correlation coefficients $(r)$ between \% liver fat, liver function tests, metabolic blood tests and BMI ( $\left.{ }^{*} \mathrm{p}<0.05\right)$

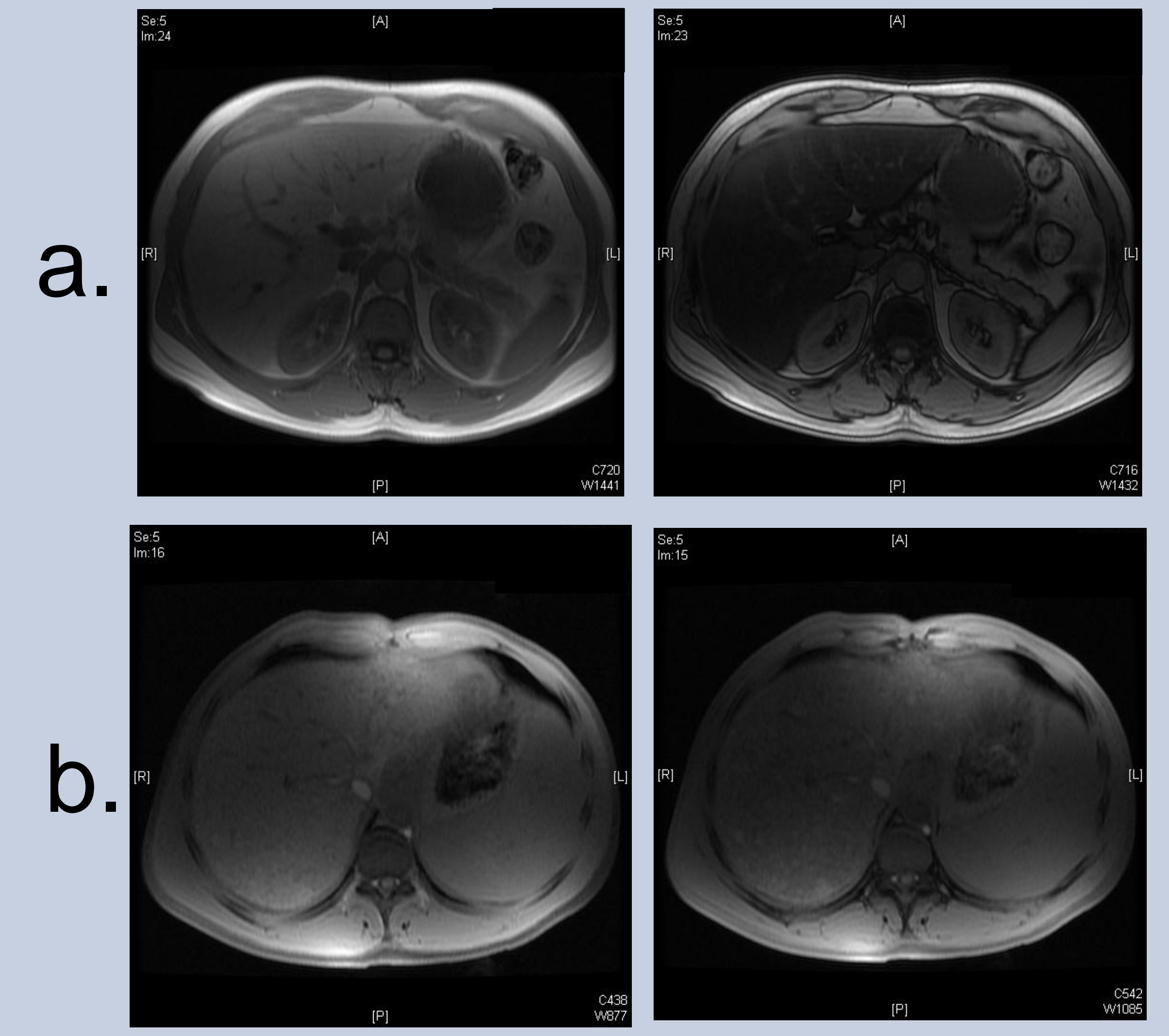

Figure 1:

In-phase (L) and out of phase (R) MR images of the liver in patients with lipodystrophy; a PPARG mutation $\%$ liver fat $=29 \%$, no other abnormal features b) 28 year male with a BSCL2 mutation $\%$ liver fat $=6 \%$, scan features consistent with liver cirrhosis and fibrosis on liver biopsy a) 56 year female with

\section{Discussion}

As expected the majority (84\%) of patients with lipodystrophy/ and or SIR of unknown cause had a percentage liver fat of $>5 \%$, signifying significant NAFLD in those patients. Serial MRI or MRS is useful in assessing response to interventions such as leptin therapy and dietary changes in individual patients. It is important to note however that a normal MRI or MRS \% liver fat result does not exclude the presence of significant NAFLD and may even signify the presence of fibrosis in some cases, although this is yet to be formally proven. 